TRANSACTIONS OF THE

AMERICAN MATHEMATICAL SOCIETY

Volume 350, Number 4, April 1998, Pages 1395-1406

S 0002-9947(98)02106-0

\title{
EXTREME POINTS OF THE DISTANCE FUNCTION ON CONVEX SURFACES
}

\author{
TUDOR ZAMFIRESCU
}

\begin{abstract}
We first see that, in the sense of Baire categories, many convex surfaces have quite large cut loci and infinitely many relative maxima of the distance function from a point. Then we find that, on any convex surface, all these extreme points lie on a single subtree of the cut locus, with at most three endpoints. Finally, we confirm (both in the sense of measure and in the sense of Baire categories) Steinhaus' conjecture that "almost all" points admit a single farthest point on the surface.
\end{abstract}

\section{INTRODUCTION}

Let $S$ be a closed convex surface, i.e. the boundary of an open bounded convex set, in the 3-dimensional Euclidean space. We denote by $\rho$ the intrinsic metric of $S$, and for $x \in S$ let $\rho_{x}: S \rightarrow \mathbb{R}$ be defined by $\rho_{x}(y)=\rho(x, y)$. In this paper we investigate the extrema of this function $\rho_{x}$.

By $C(x)$ we denote the cut locus of $x$, which is the set of all points $y \in S \backslash\{x\}$ such that no segment, i.e. shortest path, from $x$ to $y$ can be extended as a segment beyond $y$. It is known that $C(x)$ is a tree, which means that any two of its points are joined by a unique Jordan arc in $C(x)$. By $E(x)$ we denote the set of all endpoints of the tree $C(x)$, which are the points whose deletion does not disconnect $C(x)$.

The space $\mathcal{S}$ of all closed convex surfaces in $\mathbb{R}^{3}$ endowed with the well-known Pompeiu-Hausdorff metric is a Baire space.

On compact Riemannian surfaces, any cut locus is compact and has Hausdorff dimension 0 or 1 [13], [8]. The situation is more complicated for arbitrary convex surfaces. Then the cut locus need not be closed; it can even be residual on the surface.

If the cut locus is dense in $S$ - and we proved in 1982 that this holds for most convex surfaces in the sense of Baire categories [20] - then it must have infinite length, which immediately follows from its arcwise connectedness. Independently, the existence of convex surfaces with nonrectifiable cut loci was also shown by Shiohama and Tanaka in [17].

With possibly so large cut loci, it is interesting that the distribution of the relative maxima of $\rho_{x}$ on $C(x)$ is always very nice: they all lie on a single $Y$-tree, i.e. a tree with at most three endpoints, in $C(x)$. On the same Y-tree also lie all relative minima of $\left.\rho_{x}\right|_{C(x)}$ which are not endpoints of $C(x)$. In fact, all critical points of $\rho_{x}$ lie on that Y-tree, except for the case of surfaces $S$ of a very particular type. This is one of the main results of this paper. It completes our earlier result

Received by the editors April 17, 1996.

1991 Mathematics Subject Classification. Primary 52A15, 53C45.

(C)1998 American Mathematical Society 
saying that all absolute maxima of $\rho_{x}$ (if there are more than one) lie on a single Jordan subarc of $C(x)$ [26].

The second main result is the answer to a question of $\mathrm{H}$. Steinhaus: Must $\rho_{x}$ have a single absolute maximum for many points $x$ ?

The paper is organized as follows. After this introductory section there are two sections contributing to the motivation for the first main result: the first of them shows that even compact subsets of the cut locus may have infinite length, contrary to a result of Hebda in the Riemannian case; the second shows that on many convex surfaces there are infinitely many extrema of $\rho_{x}$. Finally, the last two sections contain the main results described above.

Let $S$ be a convex surface in $\mathbb{R}^{3}$. A geodesic is the image of an interval $I \subset$ $\mathbb{R}$ through a continuous mapping $c: I \rightarrow S$, such that every point in $I$ has a neighbourhood in $I$ on which $c$ is an isometry. If $I=\mathbb{R}$ and $c$ is periodic then $c(I)$ is called a closed geodesic. If $I$ is a compact interval $[a, b]$ then $c(I)$ is called a geodesic arc; if, moreover, $c(a)=c(b)$ then $c(I)$ is said to be a closed geodesic arc at $c(a)$.

A tree with four endpoints will be called an $\mathrm{H}$-tree.

We denote by $\mu_{\alpha}$ the $\alpha$-dimensional Hausdorff measure.

An open connected set is called a domain.

For any Borel set $D \subset S$, let $\omega(D)$ denote its curvature (see [1], p. 207).

The set of points in $S$ joined by at least two segments with $x \in S$ is denoted by $C_{x}$. Obviously, $C_{x} \subset C(x)$.

We denote by $M_{x}$ the set of all relative maxima of $\rho_{x}$, by $F_{x}$ its subset of all absolute maxima of $\rho_{x}$, and by $Q(J)$ the set of all relative minima of $\left.\rho_{x}\right|_{J}$, where $J$ is an arbitrary Jordan subarc of $C(x)$, minus its two endpoints. Then $Q_{x}=\bigcup_{J} Q(J)$ includes the set of all relative minima of $\left.\rho_{x}\right|_{C(x) \backslash E(x)}$. Clearly, all these are subsets of $C(x)$.

Let $E \subset S$ be the set of all endpoints of $S$, i.e. points not interior to any geodesic.

We shall make use of the set $\mathcal{S}_{2}$ of all surfaces $S \in \mathcal{S}$ possessing a point $x \in S$ with disconnected $M_{x}$. This set is obviously open in $\mathcal{S}$.

In any metric space $(\mathcal{M}, \delta)$, if $x \in \mathcal{M}$ and $\varepsilon>0$, then $B(x, \varepsilon)=\{y \in \mathcal{M}$ : $\delta(x, y)<\varepsilon\}$ and $K(x, \varepsilon)=\{y \in \mathcal{M}: \delta(x, y)=\varepsilon\}$.

\section{NonReCtifiable COMPACT SUbSETS OF THE CUT LOCUS}

Back in 1935/6 S. B. Myers [13], [14] investigated the cut locus on complete 2-dimensional Riemannian manifolds. He proved that in such a manifold each connected component of the cut locus is a local tree. In the (general) closed convex case, the cut locus must be a tree [12]. Also, Myers showed that the cut locus is triangulable if the metric is real analytic. But H. Gluck and D. Singer [6] have produced examples of smooth metrics with nontriangulable cut loci.

The situation is worse in the general case of Aleksandrov surfaces (see [17] for a definition), as can be seen from the arbitrary convex case. Then the cut loci, which must be closed in the Riemannian case (see Hebda [8]), do not necessarily enjoy this property and can be dense on the surface. Even more pathologically, while the subset of all nodes of $C_{x}$ (points joined by at least three segments with $x$ ) can be dense, $C(x) \backslash C_{x}$ and a fortiori $E(x)$ can be residual (see [20], [22], [25]). 
The central result of Hebda [9] states that in any complete 2-dimensional $C^{\infty}$ Riemannian manifold, every compact subset of a cut locus has finite 1-dimensional Hausdorff measure. This, together with an earlier result of Hebda [8], completes the solution to Ambrose's problem in dimension 2. The situation changes when we drop the differentiability assumptions on the manifold, and Hebda's result fails in arbitrary 2-dimensional Aleksandrov spaces. J.-I. Itoh ([10], Theorem B) constructed a suitable example. We use a completely different approach showing that, in fact, most surfaces can serve as examples!

"Most" means "all, except those in a set of the first Baire category". For other geometrically relevant, sometimes curious phenomena on convex surfaces, obtained via Baire categories, see [7], [23], [28].

Lemma 1. [20]. On most closed convex surfaces, most points are endpoints.

Lemma 1 shows that most $S \in \mathcal{S}$ have the properties described in [17], Examples 2 and 4, pp. 538-540. It also shows that $C(x)$ is residual on most $S \in \mathcal{S}$, in contrast to the measure-theoretic result of Otsu and Shioya [15] implying that $\mu_{2}(C(x))=0$ on all $S \in \mathcal{S}$.

The following lemma follows from Theorem 1 in [22].

Lemma 2. On any closed convex surface $S$ and for any $x \in S$, the set $C_{x}$ is of first Baire category.

We need the following refinement of Lemma 2 in [24].

Lemma 3. Let $S \in \mathcal{S}$ and $x, y, z, u, v \in S$. (The points $u, v$ may coincide.) Let $\Sigma_{u}, \Sigma_{v}$ be segments from $x$ to $u$, respectively $v$. Let $J \subset S$ be an arc with endpoints $u$, $v$ such that $J \cup \Sigma_{u} \cup \Sigma_{v}$ separates y from $z$. If no segment from $x$ to any point in $J$ passes through $y$ or $z$, then there is a point in $J$ joined with $x$ by two segments whose union separates y from $z$.

Proof. Let $w \in J$, and denote by $J_{w}$ the subarc of $J$ from $u$ to $w$ and by $\Sigma_{w}$ a segment from $x$ to $w$.

There exists a point $w_{0} \in J$ such that, for all $w \in J_{w_{0}}$, the closed curve $\Delta_{w}=$ $\Sigma_{w} \cup\left(J \backslash J_{w_{0}}\right) \cup \Sigma_{v}$ separates $y$ from $z$, but for points $w \notin J_{w_{0}}$ arbitrarily close to $w_{0}, \Delta_{w}$ does not. Then $w_{0}$ must be joined with $x$ by two segments $\Sigma_{w_{0}}, \Sigma_{w_{0}}^{\prime}$, because any sequence of segments has a subsequence convergent to a segment, and $\Sigma_{w_{0}} \cup \Sigma_{w_{0}}^{\prime}$ separates $y$ from $z$.

These lemmas will be useful in the proof of the main result of this section, which follows.

Theorem 1. On most closed convex surfaces, for any point $x$, in any open set there is a compact subset of $C_{x}$ of infinite 1-dimensional Hausdorff measure.

Proof. By Lemmas 1 and 2, on most closed convex surfaces $S$, most points of $S$ belong to the set $E \backslash C_{x}$.

Consider a point $z \in F_{x}$ and a point $y_{0} \in E \backslash\left(C_{x} \cup\{x\} \cup\{z\}\right)$ in the arbitrary open set $O \subset S$.

Choose $\eta>0$ smaller than both $\rho\left(y_{0}, x\right)$ and $\rho\left(y_{0}, z\right)$, such that $K\left(y_{0}, \eta\right)$ is a closed Jordan curve (see [1], p. 383) and $D_{0}=B\left(y_{0}, \eta\right) \subset O$. Let $\Sigma$ be the segment from $x$ to $y_{0}$.

Take a point $y^{\prime} \in B\left(y_{0}, \eta / 2\right) \backslash \Sigma$ and a segment $x y^{\prime}$. Then $\Sigma \not \subset x y^{\prime}$. Choose $y_{\infty} \in E \cap D_{0} \cap B\left(x, \rho\left(x, y_{0}\right)-\eta / 2\right)$ such that $\Sigma \cup x y^{\prime} \cup y_{0} y^{\prime}$ separates $y_{\infty}$ from $z$. 
We apply now Lemma 3 to the points $x, y=y_{\infty}, z \in F_{x}, u=y_{0}$ and $v=y^{\prime}$, and obtain a point $y_{1} \in y_{0} y^{\prime}$ joined with $x$ by two segments whose union $U$ separates $y_{\infty}$ from $z$; let $D_{1}$ be the component of $S \backslash U$ containing $y_{\infty}$.

Put $r=\rho\left(x, y_{\infty}\right)$ and $\varepsilon=\rho\left(x, y_{1}\right)-r$. We have $\varepsilon>0$, because

$$
r<\rho\left(x, y_{0}\right)-\eta / 2<\rho\left(x, y_{0}\right)-\rho\left(y_{0}, y^{\prime}\right)<\rho\left(x, y_{0}\right)-\rho\left(y_{0}, y_{1}\right) \leq \rho\left(x, y_{1}\right) .
$$

Again by Lemma 3 , on $K(x, r+\varepsilon / 2) \cap D_{1}$ there must be another point $y_{2}$ joined with $x$ by two segments determining another domain $D_{2} \subset D_{1}$ with $y_{\infty} \in D_{2}$. Continuing this way, we get the sequence $\left\{y_{n}\right\}_{n=1}^{\infty}$ with $y_{n} \in K(x, r+\varepsilon / n) \cap D_{n-1}$. This sequence must converge to $y_{\infty}$. Indeed, let $y^{\prime}$ be a limit point of $\left\{y_{n}\right\}_{n=1}^{\infty}$. Then $y^{\prime} \in K(x, r)$. For each $n, y_{m} \in \overline{D_{n}}$ for all $m \geq n$ implies $y^{\prime} \in \overline{D_{n}}$. But $\cap_{n=1}^{\infty} \overline{D_{n}}$ has only the point $y_{\infty}$ on $K(x, r)$ because every convergent subsequence of segments from $x$ to $y_{n}$ tends to a segment from $x$ to $y_{\infty}$, which has only the point $y_{\infty}$ on $K(x, r)$. Hence $y^{\prime}=y_{\infty}$.

Now choose a point

$$
z_{n} \in E \cap B(x, r) \cap D_{n} \backslash D_{n+1} .
$$

The Jordan arc in the tree $C(x)$ from $y_{n}$ to $z_{n}$ has a subarc $J_{n}$ from $y_{n}$ to some point of $K(x, r)$. Clearly, $\mu_{1} J_{n} \geq \varepsilon / n$, and $\left\{J_{n}\right\}_{n=1}^{\infty}$ converges to $\left\{y_{\infty}\right\}$. Thus, for some $n_{0} \in \mathbb{N}, J_{n} \subset O$ for all $n \geq n_{0}$, the set

$$
L=\left\{y_{\infty}\right\} \cup \bigcup_{n=n_{0}}^{\infty} J_{n} \subset C(x) \cap O
$$

is compact, and $\mu_{1} L=\infty$.

\section{INFINITE $M_{x}$ AND $Q_{x}$}

Although we saw that on many convex surfaces $C(x)$ can be dense, it is not yet clear how large the sets $M_{x}$ and $Q_{x}$ can be.

The set $F_{x}$ can be infinite, and can even have Hausdorff dimension 1, but this happens on very few, special surfaces only. (See [24], [26] for more details on $F_{x}$.) When passing to the sets $M_{x}$ and $Q_{x}$ the situation changes. We shall see here that on surfaces from a second category set, they are both infinite.

The following follows from Lemma 1 in [26] (see also Lemma 1 in [24]).

Lemma 4. Let $S$ be a convex surface. If $x, y, z \in S$ are distinct points, $\Sigma_{y}, \Sigma_{y}^{\prime}$ are distinct segments from $x$ to $y$ and $\Sigma_{z}, \Sigma_{z}^{\prime}$ are distinct segments from $x$ to $z$, then there exists a domain $\Delta$ with boundary $\Sigma_{y} \cup \Sigma_{y}^{\prime} \cup \Sigma_{z} \cup \Sigma_{z}^{\prime}$ and a Jordan arc $J_{y z}^{x}$ in $C_{x}$ joining $y$ with $z$. Moreover, every point in $J_{y z}^{x} \backslash\{y, z\}$ belongs to $\Delta$ and can be joined with $x$ by two segments the union of which separates $y$ from $z$.

Lemma 5. Let $S \in \mathcal{S}, x \in S$ and $y, z \in C_{x}$. If $u \in J_{y z}^{x}$ is a relative minimum of $\left.\rho_{x}\right|_{\text {int } J_{y z}^{x}}$, then $u$ is the midpoint of a closed geodesic arc at $x$, both subarcs from $x$ to $u$ are segments, and no other segments connect $x$ to $u$.

Proof. Suppose $\rho_{x}(u)$ is minimal in the neighbourhood $N=B(u, \varepsilon) \cap J_{y z}^{x}$ of $u$ and $\varepsilon<\min \{\rho(u, y), \rho(u, z)\}$. Let $\Sigma, \Sigma^{\prime}$ be the two segments from $x$ to $u$ given by Lemma 4. We only have to show that $\Sigma \cup \Sigma^{\prime}$ is a geodesic locally at $u$. Indeed, suppose for $v \in \Sigma, v^{\prime} \in \Sigma^{\prime}$, both in $B(u, \varepsilon / 2)$, there exists a path $P$ between them shorter than $v u \cup v^{\prime} u$. Then $P$ must cross $J_{y z}^{x}$ at some point $s$, and either the subpath from $v$ to $s$ is shorter than $v u$ or the one from $v^{\prime}$ to $s$ is shorter than $v^{\prime} u$. 
But in each case a path from $x$ to $J_{y z}^{x}$ shorter than $\Sigma$ is found and $s \in B(u, \varepsilon)$, in contradiction with the minimality of $\rho_{x}(u)$ in $N$.

Lemma 5 points out an interesting property of relative minima of $\left.\rho_{x}\right|_{C(x) \backslash E(x)}$ in the case of convex surfaces. It can be easily extended to any Aleksandrov surface.

The case of Riemannian manifolds is described in [11]. The theorem in [27] provides an extension to Aleksandrov spaces (see [3] for a definition) and gives more information in several directions. The following result is part of that theorem, applied to the particular case of a convex surface. We give it here for completeness.

Proposition 1. Let $S \in \mathcal{S}, x, y \in S$, and let $\mathcal{F}$ be the family of all segments from $x$ to $y$. If $y$ is a relative minimum of $\left.\rho_{x}\right|_{C(x)}$ and if $\mathcal{F}$ is disconnected, then $y$ is the midpoint of a closed geodesic arc at $x$, both subarcs from $x$ to $y$ are segments, and no other segments connect $x$ to $y$.

From Lemma 1 in [26] and Lemma 5 we deduce the following.

Lemma 6. Let $S \in \mathcal{S}$ and $x \in S$. If $y \in Q_{x}$ then $y$ is the midpoint of a closed geodesic arc at $x$, both subarcs from $x$ to $y$ are segments, and no other segments connect $x$ to $y$.

We denote by $T_{y}$ the space of all tangent directions at $y \in S$. ( $T_{y}$ is a closed Jordan curve in the unit 2-sphere.) We have $\mu_{1}\left(T_{y}\right)=2 \pi-\omega(\{y\})$.

The following lemma is part of Theorem 2 in [24], except for the use of a relative maximum of $\rho_{x}$ instead of an absolute one. The proof in [24] applies without change.

Lemma 7. If $S \in \mathcal{S}, x \in S$ and $y \in M_{x}$, then each arc in $T_{y}$ of length $\pi$ contains the tangent direction of a segment from $x$ to $y$. Thus, if $\omega(\{y\})<\pi$, then there are at least two segments from $x$ to $y$.

We also need Aleksandrov's gluing theorem ([1], p. 362) or its polyhedral variant ([1], p. 317); we reproduce here the latter. Consult [1] for details.

Lemma 8. If a 2-manifold $M$ results from gluing together several polygons with metrics of positive curvature such that the sum of the angles at vertices glued together is not larger than $2 \pi$, then the metric of $M$ is also of positive curvature.

Theorem 2. On most convex surfaces $S \in \mathcal{S}_{2}$ there exist a point $x$ and a Jordan arc $J$ in $C(x)$ containing infinitely many relative maxima of $\rho_{x}$ and infinitely many relative minima of $\left.\rho_{x}\right|_{J}$.

Proof. Let $\mathcal{S}_{m}(m \geq 3)$ be the family of all convex surfaces $S \in \mathcal{S}_{2}$ such that, for any point $x \in S$ and Jordan $\operatorname{arc} J \subset C(x),\left.\rho_{x}\right|_{J}$ has at most $m-2$ relative minima or maxima.

To prove the theorem it suffices to show that $\mathcal{S}_{m}$ is nowhere dense for all $m \geq 3$.

To this end, let $\mathcal{O} \subset \mathcal{S}_{2}$ be open and choose a polytopal surface $P^{\prime} \in \mathcal{O}$ with curvature less than $\pi$ at all vertices. There are three points $x^{\prime}, y^{\prime}, z^{\prime} \in P^{\prime}$ such that $y^{\prime}, z^{\prime} \in M_{x^{\prime}}$ on $P^{\prime}$. The point $x^{\prime}$ need not be a vertex. But the boundary $P$ of the convex hull of $P^{\prime}$ plus a point $x$ close to $x^{\prime}$ and outside $P^{\prime}$ will also have two points $y, z \in M_{x}$ on $P$, and moreover $x$ will be a vertex. By Lemma $7, y, z \in C_{x}$ and, by Lemma 4 , there is a Jordan arc $J_{y z}^{x} \subset C_{x}$ from $y$ to $z$.

Since $y, z \in M_{x}$, there is a point $v \in \operatorname{int} J_{y z}^{x}$ realizing the absolute minimum of $\left.\rho_{x}\right|_{J_{y z}^{x}}$. By Lemma 5, there is a closed geodesic arc $G$ at $x$, passing between $y$ and $z$, with $v$ as midpoint. Both subarcs of $G$ from $x$ to $v$ are segments. 
We cut $P$ along $G$ and get two pieces $P_{1}, P_{2}$. Let $x_{i}, v_{i} \in P_{i}$ and $G_{i} \subset P_{i}$ correspond to $x, v$ and $G$, respectively $(i=1,2)$. Choose $\varepsilon \leq \omega(\{x\}) /(2 m)$. Let tab be an isosceles triangle with the sides $t a, t b$ of length $\rho(x, v)$ and with the angle $\varepsilon$ between them.

By Lemma 8, we can glue together $P_{1}, P_{2}$ and $2 m$ copies $t_{i} a_{i} b_{i}(i=1,2, \ldots, 2 m)$ of tab as follows:

(i) $t_{1}, t_{2}, \ldots, t_{2 m}, x_{1}, x_{2}$ will coincide,

(ii) $a_{1}, a_{m+1}$ and $v_{1}$ will coincide,

(iii) $b_{m}, b_{2 m}$ and $v_{2}$ will coincide,

(iv) $b_{i}, b_{i+m}, a_{i+1}, a_{i+m+1}$ will coincide $(i=1, \ldots, m-1)$.

Denote by $P_{\varepsilon}$ the resulting polytopal surface. For $\varepsilon$ small enough there is a surface $P^{*} \in \mathcal{O}$ congruent to $P_{\varepsilon}$. Hence we have an isometry $i: P_{1} \cup P_{2} \cup \bigcup_{i=1}^{2 m} t_{i} a_{i} b_{i} \rightarrow$ $P^{*}$.

Now let $\mathcal{N} \subset O$ be a neighbourhood of $P^{*}$ in $\mathcal{S}_{2}$. If $\mathcal{N}$ is small enough, then, on any surface $S \in \mathcal{N}$, there are a point $x_{0}^{\prime}$ close to $x_{0}=i\left(x_{1}\right)=i\left(x_{2}\right)$ and points $a_{1}^{\prime}, a_{2}^{\prime}, \ldots, a_{m}^{\prime} \in M_{x_{0}^{\prime}}$ close to $i\left(a_{1}\right), i\left(a_{2}\right), \ldots, i\left(a_{m}\right)$; moreover $\omega(\{a\})<\pi$ for any point $a \in S$.

Hence, by applying Lemmas 7 and 4 again as before, we establish the existence of a Jordan arc $J_{a_{i}^{\prime} a_{i+1}^{\prime}}^{x_{0}^{\prime}} \subset C_{x_{0}^{\prime}}$ lying between the four segments from $x_{0}^{\prime}$ to $a_{i}^{\prime}$ and $a_{i+1}^{\prime}$, so that $J_{a_{i-1}^{\prime} a_{i}^{\prime}}^{x_{0}^{\prime}} \cap J_{a_{i}^{\prime} a_{i+1}^{\prime}}^{x_{0}^{\prime}}=\left\{a_{i}^{\prime}\right\}(i=1, \ldots, m-1)$. Then, obviously, $\rho_{x}$ has at least $m$ maxima and $m-1$ minima on the Jordan arc $\bigcup_{i=1}^{m-1} J_{a_{i}^{\prime} a_{i+1}^{\prime}}^{x_{0}^{\prime}} \subset C_{x}$.

Thus $\mathcal{S}_{m}$ is nowhere dense for every $m \geq 3$, and the theorem is proven.

\section{The Y-TREe CONTAINING $M_{x}$ AND $Q_{x}$}

In the tree $C(x)$ with its possibly infinitely many branches and uncountably many endpoints we shall define the antipodal tree of $x$, a subtree with only at most three endpoints containing both $M_{x}$ and $Q_{x}$. Again some preparatory material is needed.

A family $\mathcal{L}$ of closed geodesic arcs at $x \in S$ is called independent if for any $L \in \mathcal{L}$ one of the components of $S \backslash L$ is disjoint from all members of $\mathcal{L}$.

The union of two (possibly coinciding) segments from $x \in S$ to some other point $y \in S$ is called a digon at $x$. The point $y$ is then called the vertex of the digon.

A family $\mathcal{D}$ of distinct digons at $x$ is called independent if for any $D \in \mathcal{D}$ either

(i) the two segments of $D$ are distinct, one of the components of $S \backslash D$, say $\Delta$, is disjoint from all members of $\mathcal{D}$, and the angle of $D$ towards $\Delta$ at its vertex $y$ plus $\omega(\{y\})$ is not less than $\pi$, or

(ii) the two segments of $D$ coincide and $\omega(\{y\}) \geq \pi$ at the vertex $y$ of $D$ (in this case we write $\Delta=\emptyset$ ).

We call the family $\mathcal{D}$ of digons strongly independent if it is independent, and the curvature of some vertex is larger than $\pi$ or the two segments of some digon are distinct.

The next result, in a direction more general than Lemma 9, is given here for its own interest.

Proposition 2. Any independent family of closed geodesic arcs at some point contains at most three members. 
Proof. Consider the independent family $\mathcal{L}$, take $L \in \mathcal{L}$, and let $\Delta_{L}$ be the component of $S \backslash L$ from the definition of independence. From the definition of a geodesic arc we deduce that $L$ is an $n$-gon for some $n \in \mathbb{N}$, such that all its angles but one measure $\pi$. Only the angle $A_{L}$ at $x$ (towards $\Delta_{L}$ ) may be different from $\pi$, and is certainly different from 0 . Thus

$$
\omega\left(\Delta_{L}\right)=\pi+A_{L} .
$$

If $\mathcal{L}$ contains at least 4 members, then the sum of the curvatures of the domains $\left\{\Delta_{L}: L \in \mathcal{L}\right\}$ is larger than $4 \pi$, contradicting $\omega(S)=4 \pi$.

Lemma 9. Any strongly independent family of digons at some point contains at most three members. Any independent family of digons at some point contains at most three members or the surface is a tetrahedron, the digons consist of pairs of coinciding segments from the point to the vertices of the tetrahedron, and the curvature of each vertex equals $\pi$.

Proof. Consider the independent family $\mathcal{D}$ of digons at $x \in S$, take $D \in \mathcal{D}$, and let $\Delta_{D}$ be the component of $S \backslash D$ from the definition of independence, and $v_{D}$ the vertex of $D$. Then

$$
\omega\left(\Delta_{D} \cup\left\{v_{D}\right\}\right) \geq \pi+A_{D},
$$

where $A_{D}$ is the angle of $D$ at $x$ towards $\Delta_{D}$ if $\Delta_{D} \neq \emptyset$, or 0 otherwise.

If $\mathcal{D}$ contains at least 4 members, then from $\omega(S)=4 \pi$ it follows that $\operatorname{card} \mathcal{D}=4$ and $A_{D}=0$ for all $D$, i.e. the two segments forming each digon coincide, the curvature of each of the 4 vertices equals $\pi$, and the curvature of the complement of $\bigcup_{D \in \mathcal{D}}\left(\Delta_{D} \cup\left\{v_{D}\right\}\right)=\left\{v_{D}: D \in \mathcal{D}\right\}$ vanishes. Thus $S$ must be a tetrahedron.

If $\mathcal{D}$ is strongly independent, then $\omega\left(\Delta_{D} \cup\left\{v_{D}\right\}\right) \geq \pi$ for all $D \in \mathcal{D}$, with strict inequality for some $D \in \mathcal{D}$. Hence $\mathcal{D}$ cannot contain more than 3 members.

The following result follows from [17], Lemma 2.1, p. 545 (see also [12]).

Lemma 10. Let $S \in \mathcal{S}$ and $x \in S$, and let $A \subset C(x)$ be a Jordan arc with $y$ as an endpoint. Then $A$ has a definite direction $\gamma$ at $y$, and no segment from $y$ to $x$ has direction $\gamma$ at $y$.

Further, if the arc $\tau_{1} \tau_{2} \subset T_{y}$ is minimal such that $\gamma \in \tau_{1} \tau_{2}$ and in each of the directions $\tau_{1}, \tau_{2}$ there is a segment from $y$ to $x$, then $\mu_{1}\left(\tau_{1} \gamma\right)=\mu_{1}\left(\tau_{2} \gamma\right)$.

Before giving the main result of this section, we recall a similar result about $F_{x}$. We will use it in the next section.

Proposition 3 ([26]). If $S \in \mathcal{S}, x \in S$ and card $F_{x}>1$, then there exists a Jordan arc in $C(x)$ containing $F_{x}$.

By Proposition 3, if $x \in S$ and card $F_{x}>1$, then there exists a unique Jordan arc $J_{x} \subset C(x)$ with both endpoints in $F_{x}$ such that $F_{x} \subset J_{x}$. This arc $J_{x}$ is called the antipodal arc of $x$, and we put $J_{x}=F_{x}$ if $F_{x}$ contains a single point [26].

An extension of this description to all relative maxima of $\rho_{x}$ is contained in Theorem 3 .

Theorem 3. For any closed convex surface $S$ and point $x \in S$, there is a $Y$-tree in $C(x)$ containing both $M_{x}$ and $Q_{x}$.

Proof. Since for any 1, 2, or 3 points in a tree we can find a Y-subtree containing the points, suppose there are 4 points $a_{1}, a_{2}, a_{3}, a_{4}$ in $M_{x} \cup Q_{x}$, not contained in any Y-subtree of $C(x)$. Then the union of all $6 \operatorname{arcs}$ determined on $C(x)$ by the 4 
points is an H-tree $H$, as it is easily seen. Of course $a_{1}, a_{2}, a_{3}, a_{4}$ are endpoints of $H$.

If $a_{i} \in Q_{x}$, we join $a_{i}$ to $x$ by segments $\Sigma_{i}, \Sigma_{i}^{\prime}$. Since $a_{i} \notin E(x)$, by Lemma 6 , $L_{i}=\Sigma_{i} \cup \Sigma_{i}^{\prime}$ is a closed geodesic arc at $x$ and, simultaneously, a digon at $x$ as well.

If $a_{i} \in M_{x}$, then consider a Jordan $\operatorname{arc} A \subset H$ starting at $a_{i}$ and the space $T_{a_{i}}$ of all tangent directions at $a_{i}$. Let $\gamma \in T_{a_{i}}$ be the direction of $A$ given by Lemma 10, and let $\tau_{1} \tau_{2}$ be the minimal arc in $T_{a_{i}}$ containing $\gamma$ such that there is a segment from $a_{i}$ to $x$ in each of the directions $\tau_{1}, \tau_{2}$. By Lemma $7, \mu_{1}\left(\tau_{1} \tau_{2}\right) \leq \pi$. Let $D_{i}$ be the union of the two segments, a digon at $x$ with vertex $a_{i}$.

For every $i$, if the segments of $D_{i}$ are distinct, then the whole $\mathrm{H}$-tree $H$ is disjoint from one of the two components of $S \backslash D_{i}$, because $C(x) \cap D_{i}=\left\{a_{i}\right\}$. Let $\Delta_{i}$ be this component, and put $\Delta_{i}=\emptyset$ if the segments of $D_{i}$ coincide. Then $\Delta_{1}, \ldots, \Delta_{4}$ are pairwise disjoint. Moreover, if the segments of $D_{i}$ are distinct, then the angle of $D_{i}$ towards $\Delta_{i}$ plus $\omega\left(\left\{a_{i}\right\}\right)$ equals $\pi$ if $a_{i} \in Q_{x}$, and equals $2 \pi-\mu_{1}\left(\tau_{1} \tau_{2}\right) \geq \pi$ if $a_{i} \in M_{x}$. If the segments coincide, then $a_{i} \in M_{x}$ and $\omega\left(\left\{a_{i}\right\}\right)=2 \pi-\mu_{1}\left(\tau_{1} \tau_{2}\right) \geq \pi$.

Therefore the family $\mathcal{D}=\left\{D_{1}, \ldots, D_{4}\right\}$ is independent.

If some point $a_{i}$ belongs to $Q_{x}$, then $\Sigma_{i} \neq \Sigma_{i}^{\prime}$ by Lemma 6 , and the family $\mathcal{D}$ is strongly independent, contradicting Lemma 9.

If all points $a_{i}$ belong to $M_{x}$, then, by Lemma $9, S$ is a tetrahedron, there is only one segment $\Sigma$ from $x$ to $a_{1}$, and $\omega\left(\left\{a_{1}\right\}\right)=\pi$. But in this case consider a point $a^{\prime}$ close to $a_{1}$, such that the direction of the segment $a_{1} a^{\prime}$ is orthogonal in $T_{a_{1}}$ to the direction of $\Sigma$. This point is clearly farther than $a_{1}$ from $x$, contradicting $a_{1} \in M_{x}$.

Hence all points of $M_{x} \cup Q_{x}$ lie in a Y-subtree of $C(x)$.

In analogy to the antipodal arc we define, for any closed convex surface $S$ and point $x \in S$, the antipodal tree $Y_{x}$ of $x$ as the minimal (with respect to inclusion) Y-tree in $C(x)$ including both $M_{x}$ and $Q_{x}$. The existence of $Y_{x}$ follows from Theorem 3, and its uniqueness is obvious.

The antipodal tree always includes the antipodal arc, and can itself be a Jordan arc or a single point.

A point $y \in S$ is called critical with respect to $x$ (and $\rho_{x}$ ) if for any tangent direction $\tau \in T_{y}$ there is a segment from $x$ to $y$ with direction $\tau^{\prime}$ in $y$ such that $\mu_{1}\left(\tau \tau^{\prime}\right) \leq \pi / 2$ (see e.g. [4], p. 2).

Lemmas 6 and 7 imply that all points in $Q_{x} \cup M_{x}$ are critical. The above arguments, mainly using Lemma 9, show the following about the set of all points critical with respect to $x$.

Theorem 4. For any closed convex surface $S$ and point $x \in S$ either there is a $Y$-tree in $C(x)$ containing all points critical with respect to $x$, or $S$ is a tetrahedron with curvature $\pi$ at every vertex.

In the exceptional case appearing in Theorem 4 , if $x$ is interior to an edge of the tetrahedron, then $C(x)$ is an $\mathrm{H}$-tree whose endpoints, which are the vertices of the tetrahedron, are all critical with respect to $x$.

\section{Points With UNIQUe FARTHEST POINTS}

In their very nice book ([5], p. 44), Croft, Falconer and Guy write: "We take ... a ... convex surface $C$ in $\mathbb{R}^{3}$... Steinhaus ... asked ... what can be said qualitatively about the set of all "farthest points" from a point $x$. Examples $\ldots$ show that it 
need not be connected. Must this set be a single point for "almost all" $x$ on $C$ ?" Our aim here is to answer the last question.

We shall confirm Steinhaus' guess about the smallness of the set of all $x$ for which $F_{x}$ has more than a single point. This will be done with the help of the notion of porosity, which has the advantage of implying smallness in both senses, that of measure and that of Baire categories.

In any metric space $(\mathcal{M}, \delta)$, for example on a surface $S \in \mathcal{S}$, the set $M$ is porous if for each $x \in M$, there exist a number $\alpha>0$ and a point $y$ arbitrarily close to $x$ such that $B(y, \alpha \delta(x, y)) \cap M=\emptyset$. Any countable union of porous sets in $\mathcal{M}$ is then called $\sigma$-porous.

We say that any complement of a $\sigma$-porous set in the non- $\sigma$-porous space $\mathcal{M}$ contains nearly all points of $\mathcal{M}$.

In Euclidean spaces, by a version of Lebesgue's density theorem (for example Theorem 10.2 of [16], p. 129), every porous set has Lebesgue measure zero (see Zajíček's Remark 2.9 in [19], p. 318), and therefore a set containing nearly all elements is large from both the measure and the category points of view. But the complement of a null set of first category may well not contain nearly all elements, as Zajíček [19] proved.

From this it immediately follows that, on any $S \in \mathcal{S}$, if $M \subset S$ is $\sigma$-porous, then $\mu_{2}(M)=0$ and $M$ is of first Baire category.

For more information on porosity, see [19]; for applications to convexity, see [21].

At least for this section, familiarity with Aleksandrov's book [1] (or Busemann's book [2]) would be of considerable help.

Theorem 5. On any closed convex surface $S$, for nearly all points $x \in S, F_{x}$ contains a single point.

Proof. Suppose $x \in S$ and $F_{x}$ contains at least two points. Let $J_{x}$ be the antipodal arc defined right after Proposition 3, and $y, z$ its endpoints. Let $0<\varepsilon<\rho(y, z) / 4$. We consider a domain $D$ with the closed Jordan curve $J$ as boundary, such that

1) $x$ belongs to $D$,

2) $\operatorname{diam} D<\varepsilon$, and

3) no point of $F_{u}$ is at distance larger than $\varepsilon$ from $F_{x}$ if $u \in D \cup J$.

Then $J$ separates $x$ from both $y$ and $z$, because

$$
\rho(x, y)=\rho(x, z)=\frac{\rho(x, y)+\rho(x, z)}{2} \geq \frac{\rho(y, z)}{2}>\varepsilon .
$$

Also, there is no segment from any point in $J$ to $z$ passing through $y$, because otherwise its length would be at least $\rho(x, y)-\varepsilon+\rho(y, z)$ and therefore larger than $\rho(x, y)+\varepsilon$, which is absurd.

We now define an $\operatorname{arc} A$ starting at $x$. If the point $x$ is interior to a segment $\Sigma$ starting at $z$, consider an interior point $x^{\prime}$ of $\Sigma$ such that $x \in x^{\prime} z$ and $x x^{\prime} \subset D$, and then take $A=x x^{\prime}$. Suppose now $x$ is not interior to any segment starting at $z$. Let $\Sigma_{z}, \Sigma_{z}^{\prime}$ be (possibly coinciding) segments from $x$ to $z$ such that the component $\Delta$ of $S \backslash\left(\Sigma_{z} \cup \Sigma_{z}^{\prime}\right)$ containing $y$ is minimal (by inclusion). By Lemma 3, one of the components of $J \backslash\left(\Sigma_{z} \cup \Sigma_{z}^{\prime}\right)$ contains the vertex $x^{\prime \prime}$ of a digon at $z$ which separates $x$ from $y$. Lemma 4 provides the Jordan arc $J_{x^{\prime \prime} x}^{z} \subset C_{z} \cup\{x\}$ joining $x^{\prime \prime}$ to $x$. We define $A$ to be a subarc of $J_{x^{\prime \prime} x}^{z}$ starting at $x$ and lying in $D$; we may subsequently need to make $A$ even smaller.

Let $z^{\prime} \in J_{x}$ be such that $0<\operatorname{diam} J_{z z^{\prime}}^{x}<\varepsilon$ (of course, $J_{z z^{\prime}}^{x} \subset J_{x}$ ) and let $w \in \bar{\Delta}$. 
For $v \in A \backslash\{x\}$ we consider the Euclidean triangle of side-lengths $\rho(x, v), \rho(v, w)$, $\rho(w, x)$, with opposite angles $\alpha, \gamma, \beta$. Then

$$
\frac{\rho(w, x)-\rho(v, w)}{\rho(x, v)}=\frac{\sin \beta}{\sin \alpha}-\frac{\sin \gamma}{\sin \alpha}=\cos \gamma+\sin \gamma \frac{\cos \alpha-1}{\sin \alpha} .
$$

When $v$ converges to $x$, then $\alpha \rightarrow 0$ and the angle $\gamma$ converges to the angle $\gamma_{w}$ between the direction of $A$ at $x$, which exists by Lemma 10, and the direction of a certain segment from $x$ to $w$. Hence

$$
\lim _{v \rightarrow x} \frac{\rho(w, x)-\rho(v, w)}{\rho(x, v)}=\cos \gamma_{w} .
$$

This is in particular true for $w=z$ and $w=z^{\prime}$. Hence, putting $\delta=\rho(x, z)-$ $\rho\left(x, z^{\prime}\right)$,

$$
\begin{aligned}
\lim _{v \rightarrow x} \frac{\rho(v, z)-\left(\rho\left(v, z^{\prime}\right)+\delta\right)}{\rho(x, v)} & =\lim _{v \rightarrow x} \frac{\rho\left(x, z^{\prime}\right)-\rho\left(v, z^{\prime}\right)}{\rho(x, v)}-\lim _{v \rightarrow x} \frac{\rho(x, z)-\rho(v, z)}{\rho(x, v)} \\
& =\cos \gamma_{z^{\prime}}-\cos \gamma_{z}>0 .
\end{aligned}
$$

Here $\gamma_{z}$ may well equal $\pi$.

While from $x$ to $z^{\prime}$ there are two segments $\Sigma_{z^{\prime}}$ and $\Sigma_{z^{\prime}}^{\prime}$, between $v$ and $z^{\prime}$ there might well be just a single segment $\Sigma_{v z^{\prime}}$ for arbitrarily small $\rho(x, v)$, tending, when $v \rightarrow x$, to $\Sigma_{z^{\prime}}$, say. Consider the surface $S^{\prime}$ obtained from $S$ by deleting the domain (not containing $z$ ) bounded by the closed Jordan curve $\Sigma_{x v} \cup \Sigma_{v y} \cup \Sigma_{y z^{\prime}} \cup \Sigma_{z^{\prime}}$, where $\Sigma_{x v}, \Sigma_{v y}, \Sigma_{y z^{\prime}}$ are segments from $x$ to $v$, from $v$ to $y$, and from $y$ to $z^{\prime}$, respectively. This compact 2-manifold with boundary admits a segment $\Sigma_{v z^{\prime}}^{\prime}$ from $v$ to $z^{\prime}$ in its intrinsic metric $\rho^{\prime}$. We claim that this segment does not lie in the boundary of $S^{\prime}$.

Indeed, on one hand, $\Sigma_{v y} \cup \Sigma_{y z^{\prime}}$ is longer than $\Sigma_{x v} \cup \Sigma_{z^{\prime}}$, because

$$
\begin{gathered}
\rho(v, y)+\rho\left(y, z^{\prime}\right) \geq \rho(x, y)-\rho(x, v)+\rho(y, z)-\rho\left(z, z^{\prime}\right) \\
>\rho(x, y)+\rho(x, v)+\rho(y, z)-3 \varepsilon>\rho(x, y)+\rho(x, v)+\varepsilon>\rho\left(x, z^{\prime}\right)+\rho(x, v) .
\end{gathered}
$$

On the other hand, the angle between $\Sigma_{x v}$ and $\Sigma_{z^{\prime}}^{\prime}$ (towards $S^{\prime}$ ) converges to the angle $\gamma_{z^{\prime}}^{*}<\pi$ between $A$ and $\Sigma_{z^{\prime}}^{\prime}$ when $v \rightarrow x$, and is therefore smaller than $\pi$ for $A$ small enough. Here, the inequality $\gamma_{z^{\prime}}^{*}<\pi$ should be explained. The two angles at $x$ between $A$ and $\Sigma_{z}$, and between $A$ and $\Sigma_{z}^{\prime}$ are, by Lemma 10, equal (to $\gamma_{z}$ ). Now, clearly, $\gamma_{z^{\prime}}^{*}<\gamma_{z} \leq \pi$. Thus, there are paths from $v$ to $z^{\prime}$ in $S^{\prime}$ shorter than $\Sigma_{x v} \cup \Sigma_{z^{\prime}}^{\prime}$. Hence neither $\Sigma_{x v} \cup \Sigma_{z^{\prime}}$ nor $\Sigma_{v y} \cup \Sigma_{y z^{\prime}}$ is a segment in $S^{\prime}$, and the claim is proved.

We analogously get

$$
\lim _{v \rightarrow x} \frac{\rho(v, z)-\left(\rho^{\prime}\left(v, z^{\prime}\right)+\delta\right)}{\rho(x, v)}=\cos \gamma_{z^{\prime}}^{*}-\cos \gamma_{z}>0
$$

where, clearly, $\gamma_{z^{\prime}}^{*} \geq \gamma_{z^{\prime}}$.

Let $k=\left(\cos \gamma_{z^{\prime}}^{*}-\cos \gamma_{z}\right) / 3$. Then, for $A$ small enough,

$$
\frac{\rho(v, z)-\left(\rho\left(v, z^{\prime}\right)+\delta\right)}{\rho(x, v)} \geq \frac{\rho(v, z)-\left(\rho^{\prime}\left(v, z^{\prime}\right)+\delta\right)}{\rho(x, v)}>2 k
$$

for any $v \in A$. Now, let $w$ be separated from $z$ by $\Sigma_{v z^{\prime}} \cup \Sigma_{v z^{\prime}}^{\prime}$, for all $v \in A$. Then a segment $\Sigma_{x w}$ from $x$ to $w$ cuts $\Sigma_{v z^{\prime}}$ or $\Sigma_{v z^{\prime}}^{\prime}$. If $\Sigma_{x w} \cap \Sigma_{v z^{\prime}}=\left\{w^{\prime}\right\}$, then

$$
\rho(x, w) \leq \rho(x, z)=\rho\left(x, z^{\prime}\right)+\delta \leq \rho\left(x, w^{\prime}\right)+\rho\left(w^{\prime}, z^{\prime}\right)+\delta,
$$

whence

$$
\rho\left(w^{\prime}, w\right) \leq \rho\left(w^{\prime}, z^{\prime}\right)+\delta
$$


and

$$
\rho(v, w) \leq \rho\left(v, w^{\prime}\right)+\rho\left(w^{\prime}, w\right) \leq \rho\left(v, z^{\prime}\right)+\delta .
$$

If $\Sigma_{x w} \cap \Sigma_{v z^{\prime}}^{\prime} \neq \emptyset$, then, analogously, $\rho(v, w) \leq \rho^{\prime}\left(v, z^{\prime}\right)+\delta$. Hence

$$
\frac{\rho(v, z)-\rho(v, w)}{\rho(x, v)}>2 k
$$

for any $v \in A$.

Every point $u \in B(v, k \rho(x, v))$ is closer to $w$ than to $z$, because

$$
\begin{gathered}
\rho(u, z)-\rho(u, w) \geq(\rho(v, z)-\rho(v, u))-(\rho(v, w)+\rho(v, u)) \\
>\rho(v, z)-\rho(v, w)-2 k \rho(x, v)=\left(\frac{\rho(v, z)-\rho(v, w)}{\rho(x, v)}-2 k\right) \rho(x, v)>0 .
\end{gathered}
$$

Therefore, for each $u \in B(v, k \rho(x, v))$, we have $F_{u} \subset B\left(J_{z z^{\prime}}^{x}, \varepsilon\right)$, where $B\left(J_{z z^{\prime}}^{x}, \varepsilon\right)=$ $\bigcup_{t \in J_{z z^{\prime}}^{x}} B(t, \varepsilon)$.

Now the rest is easy. We show that

$$
T_{n}=\left\{x \in S: \operatorname{diam} F_{x} \geq n^{-1}\right\}
$$

is porous, for any $n \in \mathbb{N}$. Indeed, if $x \in T_{n}$, consider the endpoints $y, z$ of $J_{x}$. By taking $\varepsilon$ smaller than both $\rho(y, z) / 4$ and $(3 n)^{-1}$, we found above that, for any $v \in A$ and any $u \in B(v, k \rho(x, v))$, we have $F_{u} \subset B\left(J_{z z^{\prime}}^{x}, \varepsilon\right)$; but $\operatorname{diam} B\left(J_{z z^{\prime}}^{x}, \varepsilon\right)<$ $3 \varepsilon<n^{-1}$, whence $B(v, k \rho(x, v)) \cap T_{n}=\emptyset$.

Thus the set $\bigcup_{n} T_{n}$ of all $x \in S$ with $\operatorname{diam} F_{x}>0$ is $\sigma$-porous, and this finishes the proof.

\section{OPEN PROBLEMS}

As usual, many questions remain open. We select here only a few, which we consider most interesting.

We saw that on any closed convex surface, for nearly all $x, \operatorname{card} F_{x}=1$. But not necessarily for all $x$. Put

$$
\mathcal{S}_{(n)}=\left\{S \in \mathcal{S}: \exists x \in S \text { such that } \operatorname{card} F_{x} \geq n\right\} .
$$

It is easily seen that $\mathcal{S}_{(2)}$ is of second Baire category in $\mathcal{S}$. We even believe the following.

Conjecture 1. $\mathcal{S}_{(3)}$ is of second Baire category in $\mathcal{S}$.

H. Steinhaus conjectured that, on more or less smooth closed convex surfaces, there are points $x$ and $y \in F_{x}$ joined by at least three segments [5].

C. Vîlcu [18] disproved this for arbitrary closed convex surfaces. However, his counterexample essentially possesses points of nondifferentiability.

Conjecture 2 (Steinhaus). Every closed convex surface of class $C^{1}$ admits points $x$ and $y \in F_{x}$ joined by at least three segments.

Connected to this, we have the following.

Question. Does every surface $S \in \mathcal{S}$ possess two points $x \in S$ and $y \in F_{x}$ and two segments joining them with directions $\tau_{1}, \tau_{2}$ at $y$, such that the length of both arcs into which $\tau_{1}, \tau_{2}$ divide $T_{y}$ is at most $\pi$ ?

The following problem runs into technical difficulties.

Problem. Generalize Theorem 5 to any Aleksandrov space. 


\section{REFERENCES}

[1] A. D. Aleksandrov, Die innere Geometrie der konvexen Flächen, Akademie-Verlag, Berlin, 1955. MR 17:74d

[2] H. Busemann, Convex Surfaces, Interscience Publishers, New York, 1958. MR 21:3900

[3] Y. Burago, M. Gromov and G. Perelman, A. D. Aleksandrov spaces with curvature bounded below, Russian Math. Surveys 47 (1992), no. 2, 1 - 58. MR 93m:53035

[4] J. Cheeger, M. Gromov, C. Okonek and P. Pansu, Geometric Topology: Recent Developments, Lecture Notes in Math. 1504, Springer-Verlag, Berlin, 1991. MR 92m:53001

[5] H. T. Croft, K. J. Falconer and R. K. Guy, Unsolved problems in geometry, SpringerVerlag, New York, 1991. MR 92c:52001

[6] H. Gluck, D. Singer, Scattering of geodesic fields I, Ann. Math. (2) 108 (1978) 347-372. MR 80c:53046

[7] P. Gruber, Baire categories in convexity, in: P. Gruber, J. Wills (eds), Handbook of Convex Geometry, Elsevier Science, Amsterdam, 1993, 1327-1346. MR 94i:52003

[8] J. Hebda, Parallel translation of curvature along geodesics, Trans. Amer. Math. Soc. 299 (1987) 559-572. MR 88d:53035

[9] J. Hebda, Metric structure of cut loci in surfaces and Ambrose's problem, J. Differential Geometry 40 (1994) 621-642. MR 95m:53046

[10] J.-I. Itoh, The length of a cut locus on a surface and Ambrose's problem, J. Differential Geometry 43 (1996) 642-651. MR 97i:53038

[11] S. Kobayashi, On conjugate and cut loci, Global Differential Geometry 27 (1989) 140-169.

[12] J. Kunze, Der Schnittort auf konvexen Verheftungsflächen, Berlin, Deutscher Verlag der Wissenschaften 1969. MR 42:6760

[13] S. B. Myers, Connections between differential geometry and topology I: Simply connected surfaces, Duke Math. J. 1 (1935) 376-391.

[14] S. B. Myers, Connections between differential geometry and topology II: Closed surfaces, Duke Math. J. 2 (1936) 95-102.

[15] Y. Otsu, T. Shioya, The Riemannian structure of Alexandrov spaces, J. Differential Geometry 39 (1994) 629-658. MR 95e:53062

[16] S. Saks, Theory of the Integral (2nd Ed.), Dover Publications, 1964. MR 29:4850

[17] K. Shiohama, M. Tanaka, Cut loci and distance spheres on Alexandrov surfaces, Actes de la Table Ronde de Géométrie Différentielle (Luminy, 1992), Sém. Congr., vol. 1, Soc. Math. France, Paris, 1996, pp. 531-559. CMP 97:06

[18] C. Vîlcu, private communication.

[19] L. Zajíček, Porosity and $\sigma$-porosity, Real Analysis Exch. 13 (1987-88) 314-350; 14 (198889), 5. MR 89e:26009; MR 89m:26008

[20] T. Zamfirescu, Many endpoints and few interior points of geodesics, Inventiones Math. 69 (1982) 253-257. MR 84h:53088

[21] T. Zamfirescu, Porosity in convexity, Real Analysis Exch. 15 (1989-90) 424-436. MR 91e:52002

[22] T. Zamfirescu, Conjugate points on convex surfaces, Mathematika 38 (1991) 312-317. MR 93e:52005

[23] T. Zamfirescu, Baire categories in convexity, Atti Sem. Mat. Fis. Univ. Modena 39 (1991) 139-164. MR 92c:52002

[24] T. Zamfirescu, On some questions about convex surfaces, Math. Nachrichten 172 (1995) 313-324. MR 96e:52004

[25] T. Zamfirescu, Points joined by three shortest paths on convex surfaces, Proc. Amer. Math. Soc. 123 (1996) 3513-3518. MR 96a:52001

[26] T. Zamfirescu, Farthest points on convex surfaces, Math. Zeitschrift, to appear.

[27] T. Zamfirescu, Closed geodesic arcs in Aleksandrov spaces, Rend. Circolo Mat. Palermo Suppl. 50 (1997), 425-430.

[28] C. Zong, Strange Phenomena in Convex and Discrete Geometry, Springer-Verlag, New York, 1996. CMP 97:03

Fachbereich Mathematik, Universität Dortmund, 44221 Dortmund, Germany

E-mail address: tudor.zamfirescu@mathematik.uni-dortmund.de 